\title{
EVOLUTIONARY OPTIMIZATION OF INTERVAL MATHEMATICS-BASED DESIGN OF A TSK FUZZY CONTROLLER FOR ANTI-SWAY CRANE CONTROL
}

\author{
JAROSŁAW SMOCZEK \\ Faculty of Mechanical Engineering and Robotics \\ AGH University of Science and Technology, al. Mickiewicza 30, 30-059 Kraków, Poland \\ e-mail: smoczek@agh.edu.pl
}

\begin{abstract}
A hybrid method combining an evolutionary search strategy, interval mathematics and pole assignment-based closed-loop control synthesis is proposed to design a robust TSK fuzzy controller. The design objective is to minimize the number of linear controllers associated with rule conclusions and tune the triangular-shaped membership function parameters of a fuzzy controller to satisfy stability and desired dynamic performances in the presence of system parameter variation. The robust performance objective function is derived based on an interval Diophantine equation. Thus, the objective of a fuzzy logic-based control scheme is to place all the closed-loop control system characteristic polynomial coefficients within desired intervals. The reproduction process in the proposed Evolutionary Algorithm (EA) is based on the arithmetical crossover, uniform and non-uniform mutation along with gene deletion/insertion mutation ensuring a diversity of genomes sizes, as well as a diversity in the parameter space of membership functions. The proposed algorithm was implemented to design a fuzzy logic-based anti-sway crane control system taking into consideration the rope length and the mass of a payload variation. The results of experiments conducted using the EA for different conditions assumed for system parameter intervals and desired closed-loop system performances are compared with results achieved using the iterative procedure which is also described in the paper.
\end{abstract}

Keywords: interval mathematics, pole placement method, evolutionary algorithm, fuzzy logic, TSK controller, anti-sway crane control.

\section{Introduction}

Interval mathematics (Young, 1931; Warmus, 1956; Moore, 1966) provides useful tools for robust control system synthesis and stability analysis taking into consideration the system parameter uncertainty. The uncertain system is frequently represented by a continuous-time model with interval parameters that allows designing a robust controller through combining the interval analysis of closed-loop system performances and classic methods of controller synthesis. Numerous authors, frequently inspired by Kharitonov's theorem (Kharitonov, 1978), studied the problem of robust controller design in the presence of system parameter variations (Dahleh et al., 1993; Chapellat et al., 1994; Mallan et al., 1997). Some practical techniques of designing robust control schemes are based on iterative methods (McNichols and Fadali, 2003), modal controllers synthesis (Bańka et al., 2013), methods derived based on Lyapunov stability theory (Zubowicz and Brdyś, 2013), as well as soft computing techniques, e.g., Genetic Algorithms (GAs) (Hsu et al., 2007) and artificial neural networks (Lee et al., 2002) applied to tune linear controller parameters in terms of acceptable ranges for phase and gain margins. In this paper, EA-based synthesis of a robust TSK (Takagi and Sugeno, 1985; Sugeno and Kang, 1988) fuzzy controller which places the coefficients of a closed-loop characteristic polynomial within desired intervals is proposed and addressed to the problem of an anti-sway crane control.

The automation of crane operations is very important owing to the necessity of ensuring safety and efficiency of the transportation process, which is involved by requirements of enhancing the productivity of manufacturing processes (Smalko and Szpytko, 2009; Szpytko and Wozniak, 2007). Those requirements motivate the development and implementation of control solutions which are robust to the rope length and the mass 
of payload variations, and face up the following problem: transfer a payload as fast as possible from point to point with precise positioning at a final point and reduction of sway of a payload suspended at the end of a rope.

The best known industrial applications addressing this problem are open-loop control systems applying mostly input shaping techniques (Singer et al., 1997; Karajgikar et al., 2011), which generally rely on calculating pulse amplitudes and the time location in regard to the natural frequency of a pendulum, which unfortunately varies in relation to the system parameters (rope length and mass of a payload). In some research works the problem under consideration is solved using time-optimal control theory (Sakawa and Shindo, 1982; Auernig and Troger, 1987) combining also feedback control schemes for the desired motion trajectory tracking (Moustafa, 2001; Fang et al., 2012). Other approaches are based on an indirect adaptive control scheme, Lyapunov techniques employed for state-feedback controller design, gain-scheduling, linear quadratic Gaussian and adaptive pole-placement control schemes (Hyla, 2012).

Furthermore, soft computing techniques, especially fuzzy logic, are widely employed to the problem considered. Moon et al. (1996) applied fuzzy logic to perform an optimal control scheme, while Liu et al. (2005) incorporated a fuzzy system into a sliding mode control strategy. Linguistic-rule-based fuzzy controllers are reported by Benhidjeb and Gissinger (1995), Mahfouf et al. (2000), Yi et al. (2003) and Chang (2006), and proposed for tuning gains of a PID controller by Li and Yu (2012) or Solihin et al. (2010).

Some researchers adopted off-line or on-line techniques to design or tune fuzzy rule-based controllers. Trabia et al. (2008) proposed three fuzzy controllers with Mamdani-type rules used independently to control the crane motion, hoisting and the sway angle of a payload, and the method based on the inverse dynamic for calculating the ranges of fuzzy controller input intervals within which the membership functions were distributed. Kijima et al. (1995) employed a GA to tune triangular membership functions according to the objective function which was specified based on control performances evaluated during simulation. Liu et al. (2002) proposed two fuzzy controllers of the crane position and sway angle with singleton-type rule outputs optimized during simulation by the GA according to the cost function including the settling time, the position error and the sway angle of a payload. Chang (2007) developed a two-input (position error and sway angle) fuzzy controller with Gaussian-shaped input membership functions and output fuzzy singletons, both tuned on-line using a gradient technique. Kang et al. (1999) as well as Smoczek and Szpytko (2008) employed a TSK fuzzy switching scheme of linear controllers determined at selected operating points. Oh et al. (2004) estimated scaling factors of the TSK fuzzy-type PID controller by using a hard c-means clustering method, an artificial neural network and regression polynomials. Sadati and Hooshmand (2006) utilized a clustering method to select the operating points for a fuzzy scheduler used in tower crane control.

Other examples of soft computing approaches to the crane control problem are based on neurocontrollers tuned on-line using a backpropagation method (Mendez et al., 1999) or trained by the GA (Nakazono et al., 2007), a cerebellar model articulation controller representing a TSK fuzzy PD-type controller with a fixed number of fuzzy rules (Yu et al., 2011), and GA-based time-optimal (Kimiaghalam et al., 1999), feedforward (Kimiaghalan et al., 2002), or heuristically designed (Filipic et al., 1999) anti-sway strategies.

Most fuzzy logic-based approaches to the anti-sway crane control problem described in the literature are linguistic rule-based strategies. The proposed evolutionary fuzzy clustering or artificial neural network-based techniques of designing a fuzzy controller are only adapted to tune the membership function shapes or parameters of rule conclusions for the assumed number of fuzzy rules or sets, and involve a set of training data obtained from simulations or experiments conducted on models or real objects. The robustness of a crane control system is also frequently analysed taking into account only the rope length variation. In the previous works, Smoczek and Szpytko (2010) proposed an iterative procedure and an evolutionary algorithm (Smoczek and Szpytko, 2011) to design a TSK fuzzy controller with respect to the rope length and mass of a payload variation. However, the proposed approaches were adapted to design a fuzzy control scheme based on the objective function relating only to the acceptable maximum value of the system response overshoot.

In this paper, a hybrid method combining an evolutionary-based searching strategy, interval analysis and the pole placement method is applied to design a TSK fuzzy controller which places the coefficients of the closed-loop control system characteristic polynomial within desired intervals. The paper describes a reproduction strategy which allows minimizing the fuzzy sets and tuning the parameters of membership functions of a TSK controller with respect to the control performance requirements taking into consideration the rope length and the payload variation. The paper proposes also an iterative procedure of designing a TSK controller which has been developed based on the method described by Smoczek and Szpytko (2010). Both methods allow to design robust fuzzy controllers. However, the results of experiments conducted for different conditions assumed for desired closed-loop system performances proved that the EA results in a number of fuzzy sets and rules of a TSK controller required to satisfy an acceptable range of 
closed-loop system performance deterioration specified in the form of desired intervals of closed-loop characteristic polynomial coefficients.

The paper is organized as follows. Section 2 describes a fuzzy logic-based control scheme for a planar model of a crane and the conditions assumed to design a TSK controller. In Section 3, the EA used to design a TSK fuzzy controller is presented. In Section 4, simulation examples are provided to show the effectiveness of the proposed method for anti-sway crane control system design in a bounded range of parameter variations. The results obtained by applying the EA are also compared with those derived from the iterative procedure described in Section 4. Section 5 delivers the final conclusions.

\section{Fuzzy logic-based control scheme}

The system under consideration is a planar model of a crane transferring a payload with mass $m$ suspended at the end of a rope with length $l$ (Fig. 1). The model is considered to be linear with varying parameters $l$ and $m$. The motion equations of this system

$$
\left\{\begin{array}{c}
(M+m) \ddot{x}+m l \ddot{\alpha} \cos \alpha-m l \dot{\alpha}^{2} \sin \alpha=u, \\
m \ddot{x} \cos \alpha+m l \ddot{\alpha}+m g \sin \alpha=0
\end{array}\right.
$$

were derived from Lagrange's second law type equation and after linearization (assuming $\cos \alpha \cong 1, \sin \alpha \cong \alpha$, $\dot{\alpha}^{2} \cong 0$ ) they were written down as two continuous transfer functions

$$
\begin{aligned}
& \frac{\alpha(s)}{U(s)}=\frac{-K}{s^{2}+\omega_{n}^{2}}, \\
& \frac{X(s)}{\alpha(s)}=\frac{-l s^{2}-g}{s^{2}},
\end{aligned}
$$

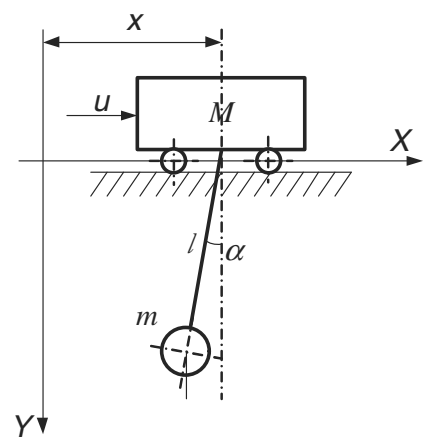

Fig. 1. Planar model of a crane, where $M, m, l, u$ and $\alpha$ are respectively the masses of a crane and payload, the rope length, the controlling signal corresponding to the control force acting on the crane, and the sway angle of the payload. where $K=1 / M l$,

$$
\omega_{n}=\sqrt{\left(1+\frac{m}{M}\right) \frac{g}{l}}
$$

is the natural (not damped) pulsation, $g=9.81 \mathrm{~m} / \mathrm{s}^{2}$ is the gravity acceleration. The adaptive control scheme can be based on a set of linear controllers determined at selected operating points. Taking into account that the transfer function (2) represents a second-order astatic system, the two PD controllers-based crane position and sway angle of a payload control algorithm can be presented as follows:

$$
U(s)=\left(k_{1}+k_{2} s\right) E(s)+\left(k_{3}+k_{4} s\right) \alpha(s),
$$

where $e=x_{r}-x$ is the error of the crane position $\left(x_{r}, x\right.$ are the reference signal and actual crane position, respectively), $k_{1}, k_{2}, k_{3}, k_{4}$ are the proportional and derivative gains of PD controllers. The control scheme can be elaborated based on a TSK fuzzy system with triangular-shaped membership functions, which is also called in the literature a P1-TS system to emphasize that membership functions of fuzzy sets for input variables are polynomial of the first order (Kluska, 2006; 2009). In the approach considered in this paper, the fuzzy logic-based adaptive control scheme is presented as a set of $N$ rules with conclusions representing the linear control law (4):

$$
R_{k} \text { : IF } l \text { is } \mathbf{A}_{i} \text { and } m \text { is } \mathbf{B}_{j} \text { THEN } u_{k}=\mathbf{K}_{k}^{T} \mathbf{X} \text {, }
$$

where $\mathbf{A}_{i}$ and $\mathbf{B}_{j}$ are the fuzzy sets on $l$ and $m$ input variables universe of discourse, respectively, where $i=$ $1,2, \ldots, n_{1}$ and $j=1,2, \ldots, n_{2}\left(n_{1}\right.$ and $n_{2}$ are the numbers of fuzzy sets defined for $l$ and $m$, respectively), $\mathbf{K}_{k}=\left[k_{1}, k_{2}, k_{3}, k_{4}\right]_{k}^{T}, \mathbf{X}=[e, \dot{e}, \alpha, \dot{\alpha}]^{T}, k=$ $1,2, \ldots, N$ (where $N=n_{1} \cdot n_{2}$ ).

The fuzzy sets defined in the premises of fuzzy rules correspond to the triangular membership functions
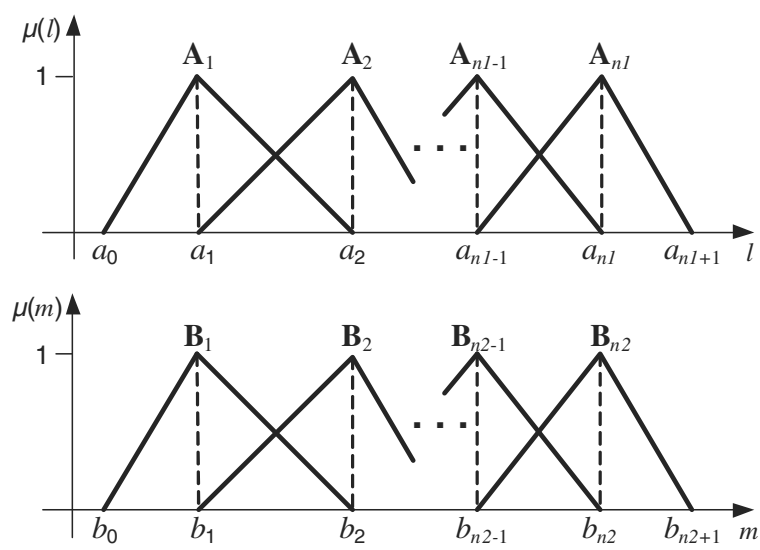

Fig. 2. Membership functions defined for fuzzy sets on the input variables $l$ and $m$. 
(Fig. 2). The membership degree of a crisp input value to the fuzzy set is calculated according to the functions

$$
\begin{aligned}
\mu_{\mathbf{A}_{i}}(l) & =\max \left(\min \left(\frac{l-a_{i-1}}{a_{i}-a_{i-1}}, \frac{a_{i+1}-l}{a_{i+1}-a_{i}}\right), 0\right), \\
\mu_{\mathbf{B}_{j}}(m) & =\max \left(\min \left(\frac{m-b_{j-1}}{b_{j}-b_{j-1}}, \frac{b_{j+1}-m}{b_{j+1}-b_{j}}\right), 0\right),
\end{aligned}
$$

where $a_{i-1} \leq a_{i} \leq a_{i+1}, b_{j-1} \leq b_{j} \leq b_{j+1}, a_{i}, b_{j}$ are the centre points of triangular membership functions of the fuzzy sets $\mathbf{A}_{i}$ and $\mathbf{B}_{j}$ (where $i=1,2, \ldots, n_{1}$ and $\left.j=1,2, \ldots, n_{2}\right)$.

The output of a fuzzy controller is calculated as the weighted average of all rules' output

$$
u=\left(\sum_{k=1}^{N} w_{k} \mathbf{K}_{k}^{T}\right)\left(\sum_{k=1}^{N} w_{k}\right)^{-1} \mathbf{X}
$$

where a rule's activation degree (firing strength) is

$$
w_{k}=\mu_{\mathbf{A}_{i}}(l) \cdot \mu_{\mathbf{B}_{j}}(m) .
$$

The problem of designing a TSK fuzzy controller consists in selecting a minimum set of operating points $\left\{a_{i}, b_{j}\right\}$ corresponding to the midpoints of triangular-shaped membership functions at which the linear controllers can be determined based on the Diophantine equation:

$$
s^{4}+\mathbf{s}\left[\begin{array}{ccccc}
0 & 0 & K l & 0 & K \\
\omega_{n}^{2} & K l & 0 & K & 0 \\
0 & 0 & K g & 0 & 0 \\
0 & K g & 0 & 0 & 0
\end{array}\right]\left[\begin{array}{c}
1 \\
k_{1} \\
k_{2} \\
k_{3} \\
k_{4}
\end{array}\right]_{k}=s^{4}+\mathbf{s P}_{k}
$$

where $\mathbf{s}=\left[s^{3}, s^{2}, s^{1}, 1\right]$, and $\mathbf{P}_{k}$ is a vector of desired coefficients of a closed-loop characteristic polynomial. Thus, the vector $\mathbf{K}_{k}$, which is defined in the conclusion of a fuzzy rule $R_{k}(5)$, can be derived from

$$
\mathbf{S}_{k} \mathbf{K}_{k}=\mathbf{P}_{k},
$$

where

$$
\mathbf{S}_{k}=\left[\begin{array}{cccc}
0 & K l & 0 & K \\
K l & 0 & K & 0 \\
0 & K g & 0 & 0 \\
K g & 0 & 0 & 0
\end{array}\right]
$$

and $\mathbf{P}_{k}$ is a vector of the nominal values of the interval coefficients vector

$$
\left[\mathbf{P}_{k}\right]=\left[\left[p_{3}\right]_{k},\left[p_{2}\right]_{k}-\omega_{n}^{2},\left[p_{1}\right]_{k},\left[p_{0}\right]_{k}\right]^{T},
$$

where $\left[p_{r}\right]_{k}=\left[p_{r}^{-}, p_{r}^{+}\right]_{k}=\left\{p_{r} \in \mathbb{R} \mid p_{r}^{-} \leq p_{r} \leq p_{r}^{+}\right\}$, $r=0,1,2,3$.

Therefore, the fuzzy logic-based control scheme satisfies the desired performances for the system parameters varying within the expected ranges $l \in\left[l^{-}, l^{+}\right]$ and $m \in\left[m^{-}, m^{+}\right]$if the condition

$$
\mathbf{S}(l, m) \mathbf{K}(l, m) \in\left[\mathbf{P}_{k}\right],
$$

is not violated for at least one interval vector (13) associated with a rule which has been activated with degree $w_{k}>0$ to interpolate the vector $\mathbf{K}(l, m)$ according to

$$
\mathbf{K}(l, m)=\left(\sum_{k=1}^{N} w_{k} \mathbf{K}_{k}\right)\left(\sum_{k=1}^{N} w_{k}\right)^{-1}
$$

\section{Evolutionary optimization of a TSK controller}

In this section the Pittsburgh-based (Smith, 1980; De Jong et al., 1993) evolutionary approach to optimize the membership function parameters and the Rule Base (RB) size is proposed. A single proposition of a TSK controller can be represented by a real-valued chromosome consisting of the triangular membership functions parameters (Fig. 2)

$$
\begin{aligned}
\mathbf{a} & =\left[a_{0}, a_{1}, \ldots, a_{i}, \ldots, a_{n_{1}}, a_{n_{1}+1}\right], \\
\mathbf{b} & =\left[b_{0}, b_{1}, \ldots, b_{j}, \ldots, b_{n_{2}}, b_{n_{2}+1}\right] .
\end{aligned}
$$

The Fuzzy Rule-Base System (FRBS) design is a searching process consisting in exploration of the solution space composed of individuals with a different chromosome size. The EA proposed in this paper is a three-stage reproduction-based strategy combining the arithmetical crossover, uniform and non-uniform mutation (Fig. 3). In each generation the reproduction process starts from group $\lambda_{1}$ of individuals to increase the population to the number $\lambda_{1}+\lambda_{2}+\lambda_{3}+\lambda_{4}$. In the first step (mutation $\mathrm{A}$ ), the genotype of the individuals selected from population $\lambda_{1}$ is changed through insertion or deletion of genes resulting in adding or removing fuzzy sets for the randomly chosen input variable. This leads to producing a small group of new individuals $\lambda_{2}$ with different sizes of the RB which are added to the population $\lambda_{1}$. The recombination and non-uniform mutation result in adding to the current population $\left(\lambda_{1}+\lambda_{2}\right)$ new individuals $\lambda_{3}$ and $\lambda_{4}$. Hence the final population size in a single generation equals $\lambda_{1}+\lambda_{2}+\lambda_{3}+\lambda_{4}$, and from this group of individuals a new population $\lambda_{1}$ is selected using the tournament method to be the parents of the next generation.

The aim of the first mutation is to bring the diversity of genome sizes into the current population through changing the number of fuzzy sets for the randomly chosen input variable. The probability of insertion $p_{I}$ or deletion $p_{D}$ of a gene depends on the average $\bar{n}$, minimum $n_{\min }$ and maximum $n_{\max }$ numbers of fuzzy sets defined for the input variable 


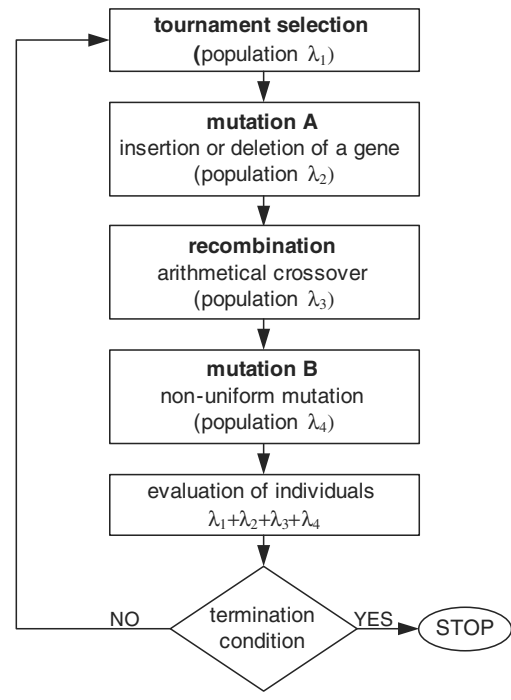

Fig. 3. EA flowchart.

$$
p_{D}=\frac{\bar{n}-n_{\min }}{n_{\max }-n_{\min }}, \quad p_{I}=1-p_{D}
$$

Fuzzy set reduction is obtained using a randomly selected method: through removing a fuzzy set or merging the randomly selected two neighboring membership functions according to the formula

$$
\begin{aligned}
& a_{i}^{\prime}=z a_{i}+(1-z) a_{i+1}, \\
& b_{j}^{\prime}=z b_{j}+(1-z) b_{j+1},
\end{aligned}
$$

where $a_{i}^{\prime}, b_{j}^{\prime}$ are the new genes of a chromosome (16) obtained through merging $a_{i}$ and $a_{i+1}$, or $b_{j}$ and $b_{j+1}$ centre points of membership functions (where $i=$ $\left.1,2, \ldots, n_{1}-1, j=1,2, \ldots, n_{2}-1\right), z$ is a uniformly distributed random number in the interval $[0,1]$.

An increase in the chromosome size is performed through uniform mutation, depending on the locus of a new gene corresponding to the midpoint of a new membership function:

$$
\begin{aligned}
{\left[a_{i-1}^{\prime}, a_{i}^{\prime}\right] } & =\left[a_{i-1}, a_{i}\right]-z\left(a_{i}-a_{i-1}\right), \quad i=1, \\
a_{i}^{\prime} & =z a_{i-1}+(1-z) a_{i}, \quad 1<i<n_{1}+1, \\
{\left[a_{i}^{\prime}, a_{i+1}^{\prime}\right] } & =\left[a_{i-1}, a_{i}\right]+z\left(a_{i}-a_{i-1}\right), \quad i=n_{1}+1,
\end{aligned}
$$

and

$$
\begin{aligned}
{\left[b_{j-1}^{\prime}, b_{j}^{\prime}\right] } & =\left[b_{j-1}, b_{j}\right]-z\left(b_{j}-b_{j-1}\right), \quad j=1, \\
b_{j}^{\prime} & =z b_{j-1}+(1-z) b_{j}, \quad 1<j<n_{2}+1, \\
{\left[b_{j}^{\prime}, b_{j+1}^{\prime}\right] } & =\left[b_{j-1}, b_{j}\right]+z\left(b_{j}-b_{j-1}\right), \quad j=n_{2}+1 .
\end{aligned}
$$

The crossover is conducted on the population $\lambda_{1}$ and the small population $\lambda_{2}$ of frequently worse individuals.
Thus, random selection of parents ensures that the crossover can be also conducted between individuals from both populations. Simultaneously, tournament selection guarantees that the recombination is performed on the best individuals $\lambda_{1}$ from the previous generation $\left(\lambda_{1}+\lambda_{2}+\right.$ $\left.\lambda_{3}+\lambda_{4}\right)$. The recombination process is performed using the arithmetical crossover method conducted on the two individuals $\mathrm{A}$ and $\mathrm{B}$ that leads to obtaining the offspring $A^{\prime}$ and $B^{\prime}$ (21). If the number of fuzzy sets for a given input variable is different in the chromosomes A and B, the crossover is performed between genes representing the closest pairs of membership function midpoints, which is illustrated in Fig. 4. The offspring A' and B' inherit the chromosome size from the parents $\mathrm{A}$ and $\mathrm{B}$, respectively:

$$
\begin{aligned}
& a_{i}^{\left.\prime{ }^{\prime}\right)}=z a_{i}{ }^{(A)}+(1-z) a_{i}{ }^{(B)}, \\
& b_{j}^{\left.\prime{ }^{\prime}\right)}=z b_{j}{ }^{(A)}+(1-z) b_{j}{ }^{(B)}, \\
& a_{i}^{\prime\left(B^{\prime}\right)}=z a_{i}{ }^{(B)}+(1-z) a_{i}{ }^{(A)}, \\
& b_{j}^{\prime\left(B^{\prime}\right)}=z b_{j}{ }^{(B)}+(1-z) b_{j}{ }^{(A)} .
\end{aligned}
$$

The last stage of the reproduction process (mutation B) is based on non-uniform mutation (Michalewicz and Janikow, 1991) conducted on a randomly chosen gene of a randomly selected chromosome. The offspring is created according to a randomly selected formula

$$
\begin{array}{r}
a_{i}^{\prime}= \begin{cases}a_{i}+\left(1-z^{\gamma}\right)\left(a_{i+1}-a_{i}\right), & i<n_{1}+1, \\
a_{i}+\left(1-z^{\gamma}\right)\left(a_{i}-a_{i-1}\right), & i=n_{1}+1,\end{cases} \\
b_{j}^{\prime}= \begin{cases}b_{j}+\left(1-z^{\gamma}\right)\left(b_{j+1}-b_{j}\right), & j<n_{2}+1, \\
b_{j}+\left(1-z^{\gamma}\right)\left(b_{j}-b_{j-1}\right), & j=n_{2}+1,\end{cases}
\end{array}
$$

or

$$
\begin{aligned}
& a_{i}^{\prime}= \begin{cases}a_{i}-\left(1-z^{\gamma}\right)\left(a_{i+1}-a_{i}\right), & i=0, \\
a_{i}-\left(1-z^{\gamma}\right)\left(a_{i}-a_{i-1}\right), & i>0,\end{cases} \\
& b_{j}^{\prime}= \begin{cases}b_{j}-\left(1-z^{\gamma}\right)\left(b_{j+1}-b_{j}\right), & j=0, \\
b_{j}-\left(1-z^{\gamma}\right)\left(b_{j}-b_{j-1}\right), & j>0,\end{cases}
\end{aligned}
$$

in which the exponent $\gamma$ of the random number $z$ equally distributed in the interval $[0,1]$ is determined based on the ratio of the number of the current generation $t$ to the maximum number of generations $t_{\max }(24)$,

$$
\gamma=\left(1-\frac{t}{t_{\max }}\right)^{\beta}
$$

where $\beta>0$ is a parameter determining the degree of dependency on the generation number (in the numerical experiments presented in Section 4 this parameter was assumed as 2).

Both mutations $\mathrm{A}$ and $\mathrm{B}$ play a significant role preventing from premature convergence of population to the suboptimal regions. The mutation $\mathrm{A}$ results in a diversity of genome sizes, while the mutation B leads to a diversity in the parameter space of rule antecedents, 


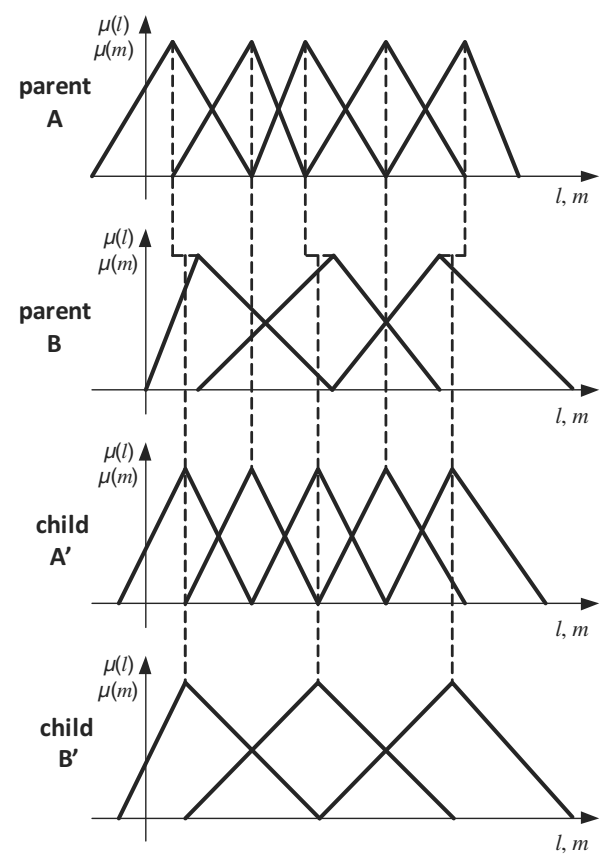

Fig. 4. Illustration of crossover between individuals which differ in the genome size.

and the conclusions. The recombination mechanism ensures fine exploration of the best promising regions of the solution space by tuning the membership function parameters.

The fitness of an individual is determined through testing the condition (14) for the most hazardous operating points corresponding to all possible combinations of the crossover points of triangular membership functions (Fig. 2), their midpoints, and the bounds of system parameter intervals $\left[l^{-}, l^{+}\right]$and $\left[m^{-}, m^{+}\right]$. Each vector $\mathbf{K}_{k}$ in a rule's conclusion (5) is derived from the system (11) for the midpoints of $N$ interval vectors of the coefficients of the desired closed-loop characteristic polynomial. The fitness of an individual is calculated as a sum of the normalized distances between the coefficients of the closed-loop system characteristic equation at the most hazardous operating points and the closest bounds of desired polynomial coefficient intervals:

$$
=\sum_{h=1}^{H} \sum_{k=1}^{N} \sum_{r=0}^{3}\left(\beta_{1} \beta_{2} \frac{\min \left(\left|p_{r}-p_{r}^{-}\right|_{k},\left|p_{r}-p_{r}^{+}\right|_{k}\right)}{\left|p_{r}^{+}-p_{r}^{-}\right|_{k}}\right)_{h},
$$

where

$$
\begin{gathered}
\beta_{1}=\left\{\begin{array}{cc}
0, & w_{k}=0, \\
1, & w_{k}>0,
\end{array}\right. \\
\left\{\begin{array}{cl}
\beta_{2}=1 & \text { if } \quad \mathbf{S}(l, m) \mathbf{K}(l, m) \in\left[\mathbf{P}_{k}\right], \\
\beta_{2}>1 & \text { if } \quad \mathbf{S}(l, m) \mathbf{K}(l, m) \notin\left[\mathbf{P}_{k}\right],
\end{array}\right.
\end{gathered}
$$

$p_{r}$ is the closed-loop characteristic polynomial coefficient, $H$ is the number of operating points at which the condition (14) is tested, $\beta_{2}$ is the penalty factor, which is $\beta_{2}>$ 1 if the condition (14) is violated (in the numerical experiments this factor was assumed to be 4 ).

\section{Simulations results}

The proposed hybrid method combining the evolutionary-based searching strategy, interval mathematics and pole assignment-based closed-loop control synthesis was applied to design a TSK fuzzy controller for performance requirements defined in the form of the desired stable poles intervals

$$
\left[s_{r}\right]_{k}=\left[s_{r}^{-}, s_{r}^{+}\right]_{k}=\left[-\omega_{n} \mp \sqrt{\frac{2 g}{l}}(1-\zeta)\right]_{k},
$$

where $\zeta$ is the parameter which determines the width of a desired stable pole interval.

Thus, a vector $\mathbf{K}_{k}$ in the conclusion of each rule $R_{k}$ (5) were determined at the operating point $\left\{a_{i}, b_{j}\right\}$ through assigning all closed-loop system poles at a nominal value of the interval (26). The EA described in Section 3, with the population composed of 48 individuals ( $\left.\lambda_{1}=12, \lambda_{2}=4, \lambda_{3}=28, \lambda_{4}=4\right)$, was employed to find the numbers of membership functions and the distribution of their parameters to satisfy the closed-loop system performances for two pairs of rope length and mass of payload intervals: $[1 \mathrm{~m}, 8 \mathrm{~m}],[10 \mathrm{~kg}, 600 \mathrm{~kg}]$ and $[1 \mathrm{~m}$, $10 \mathrm{~m}],[10 \mathrm{~kg}, 1000 \mathrm{~kg}]$. For each pair of those intervals, two experiments were conducted for assumed $\zeta=0.69$ and $\zeta=0.76$, which were used to specify an acceptable range of closed-loop system performance deterioration. Tables 1 and 2 present the results of experiments, where $n_{1}$ and $n_{2}$ are respectively the numbers of fuzzy sets determined for variables $l$ and $m, N$ is the number of fuzzy rules of the TSK fuzzy controller, $\mathbf{a}$ and $\mathbf{b}$ are the vectors (19) representing the best chromosome obtained in the last generation. The termination condition of the EA was assumed as the maximum number of generations equal to 100 .

The experiments resulted in designing TSK controllers placing the coefficients of the characteristic polynomial of the closed-loop system within the desired intervals for operating points lying within the expected intervals of the rope length and payload mass. The performances of the EA employed to find an appropriate number of fuzzy sets and tune the membership function parameters for system parameter intervals $[1 \mathrm{~m}, 8 \mathrm{~m}]$ and $[10 \mathrm{~kg}, 600 \mathrm{~kg}]$, and for $\zeta=0.69$ and $\zeta=0.76$ are illustrated in the form of the best value of the fitness function in each epoch (Fig. 5) and as the comparison of $\mathrm{RB}$ sizes of the 12 best individuals selected as the parents of the next generation (Figs. 6 and 7). The experiments 
proved the ability of the developed evolutionary strategy to optimize the number of the TSK controller's fuzzy rules. Figures 6 and 7 illustrate the influence of a mutation process (gene insertion and deletion) on the diversity of the genome size in the current population that allows finding a suitable solution of a TSK controller with a minimum number of fuzzy sets, which satisfies an acceptable range of performance deterioration. Figure 6 presents the number of individuals of population $\lambda_{1}$ representing the different RB sizes in the first 25 generations. The solutions with $20\left(n_{1}=5, n_{2}=4\right)$, $15\left(n_{1}=5, n_{2}=3\right), 10\left(n_{1}=5, n_{2}=5\right)$ and 12 $\left(n_{1}=4, n_{2}=3\right.$ ) rules dominated in the consecutive epochs until the 14th generation, while the population representing 8 rules started to growth up from the 12th epoch, completely dominating the population from the 18th epoch. Figure 7 illustrates the evolution of population during the first 40 generations in the second experiment conducted for more rigorous acceptable range of performance deterioration $(\zeta=0.76)$. During the first 13 generations the population is dominated by chromosomes representing fuzzy controllers with respectively $36\left(n_{1}=6, n_{2}=6\right), 30\left(n_{1}=6, n_{2}=5\right)$, $24\left(n_{1}=6, n_{2}=4\right)$ and $20\left(n_{1}=5, n_{2}=4\right)$ rules. From the 17 th until the 100th epoch, the best solutions are mostly represented by the RB consisting of 15 if-then rules $\left(n_{1}=5, n_{2}=3\right)$.

Examples of closed-loop system performances of the fuzzy control system designed for scheduling variables intervals $[1 \mathrm{~m}, 8 \mathrm{~m}]$ and $[10 \mathrm{~kg}, 600 \mathrm{~kg}]$ are presented

Table 1. Results of experiments obtained using the EA.

\begin{tabular}{|c|c|c|c|c|}
\hline$l$ and $m$ intervals & $\zeta$ & $n_{1}$ & $n_{2}$ & $N=n_{1} \cdot n_{2}$ \\
\hline \hline$[1,8] \mathrm{m}$ & 0.69 & 4 & 2 & 8 \\
{$[10,600] \mathrm{kg}$} & 0.76 & 5 & 3 & 15 \\
\hline$[1,10] \mathrm{m}$ & 0.69 & 5 & 3 & 15 \\
{$[10,1000] \mathrm{kg}$} & 0.76 & 6 & 3 & 18 \\
\hline
\end{tabular}

Table 2. Parameters of membership functions tuned using the EA.

\begin{tabular}{|c|c|c|}
\hline $\begin{array}{l}l \text { and } m \text { in- } \\
\text { tervals }\end{array}$ & $\zeta$ & $\begin{array}{l}\text { parameters of membership func- } \\
\text { tions }\end{array}$ \\
\hline $\begin{array}{l}{[1,8] \mathrm{m},} \\
{[10,600] \mathrm{kg}}\end{array}$ & 0.69 & 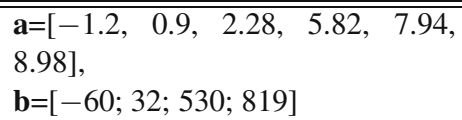 \\
\hline $\begin{array}{l}{[1,8] \mathrm{m},} \\
{[10,600] \mathrm{kg}}\end{array}$ & 0.76 & $\begin{array}{l}\mathbf{a}=[-0.13,1.06,1.85,3.26,5.00, \\
7.60,13.41], \\
\mathbf{b}=[-93,9,334,611,799]\end{array}$ \\
\hline $\begin{array}{l}{[1,10] \mathrm{m},} \\
{[10,1000] \mathrm{kg}}\end{array}$ & 0.69 & $\begin{array}{l}\mathbf{a}=[-0.45,1.04,2.00,3.41,5.82, \\
10.66,18.15], \\
\mathbf{b}=[-42,61,615,999,1646]\end{array}$ \\
\hline $\begin{array}{l}{[1,10] \mathrm{m},} \\
{[10,1000] \mathrm{kg}}\end{array}$ & 0.76 & $\begin{array}{l}\mathbf{a}=[-0.15,0.86,1.40,2.17,3.84, \\
6.09,10.68,12.68], \\
\mathbf{b}=[-13,45,348,1008,1615]\end{array}$ \\
\hline
\end{tabular}

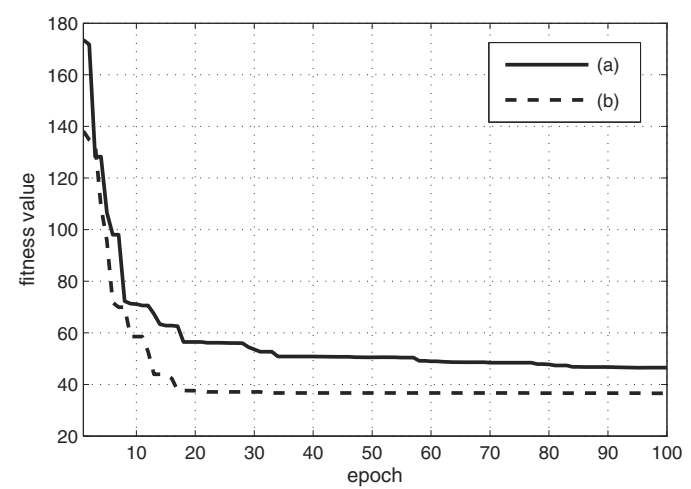

Fig. 5. Comparison of the best value of the fitness function in each epoch-experiments for parameters $[1 \mathrm{~m}, 8 \mathrm{~m}]$ [10 kg, $600 \mathrm{~kg}], \zeta=0.76$ (a), [1 m, $8 \mathrm{~m}],[10 \mathrm{~kg}, 600$ $\mathrm{kg}], \zeta=0.69(\mathrm{~b})$

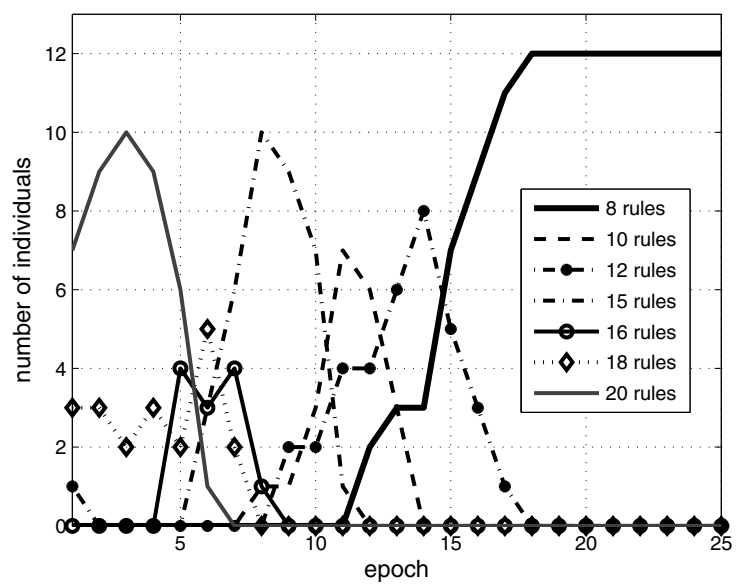

Fig. 6. Size comparison of the best individuals in the first 25 epochs-experiment for $\zeta=0.69,[1 \mathrm{~m}, 8 \mathrm{~m}],[10 \mathrm{~kg}$, $600 \mathrm{~kg}]$.

in Figs. 8 and 9 in the form of unit-step system responses (the crane position and the sway angle of the payload) at selected most hazardous operating points corresponding to the crossover points of membership functions. The solid line (a) in Fig. 8 represents the response at the operating point corresponding to the crossover points of membership functions $\mathbf{A}_{1}$ and $\mathbf{A}_{2}$, and $\mathbf{B}_{1}$ and $\mathbf{B}_{2}$ with the centre points $a_{1}=0.9 \mathrm{~m}, a_{2}=2.28 \mathrm{~m}$, $b_{1}=32 \mathrm{~kg}$ and $b_{2}=530 \mathrm{~kg}$ determined using the EA for $\zeta=0.69$ (Table 2). The dotted lines (s-) and (s+) represent the responses of a classic PD controller-based closed-loop control system designed at $\left\{a_{1}, b_{2}\right\}$ through assigning the poles at right and left bounds of the desired interval (26). The condition (14) is satisfied for the examined operating point $\left\{\left(a_{1}+a_{2}\right) / 2,\left(b_{1}+\right.\right.$ $\left.\left.b_{2}\right) / 2\right\}$, because the characteristic polynomial coefficients 


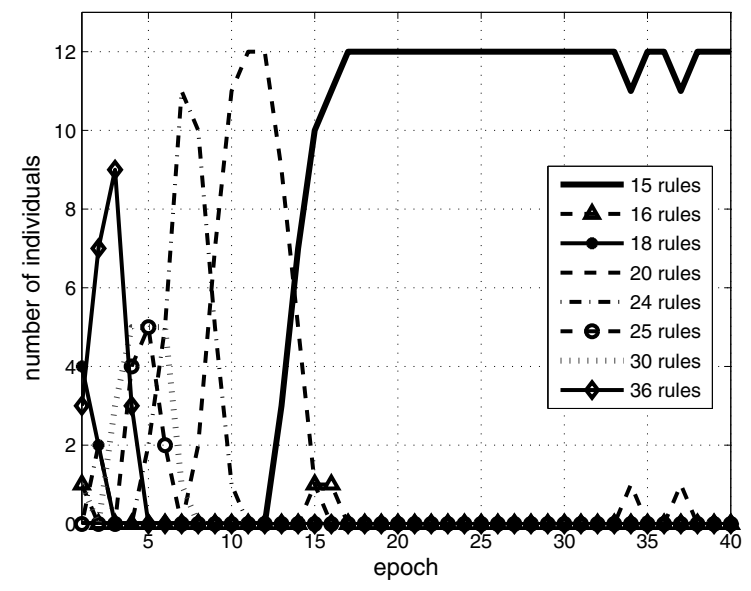

Fig. 7. Size comparison of the best individuals in the first 25 epochs-experiment for $\zeta=0.76,[1 \mathrm{~m}, 8 \mathrm{~m}],[10 \mathrm{~kg}$, $600 \mathrm{~kg}$.
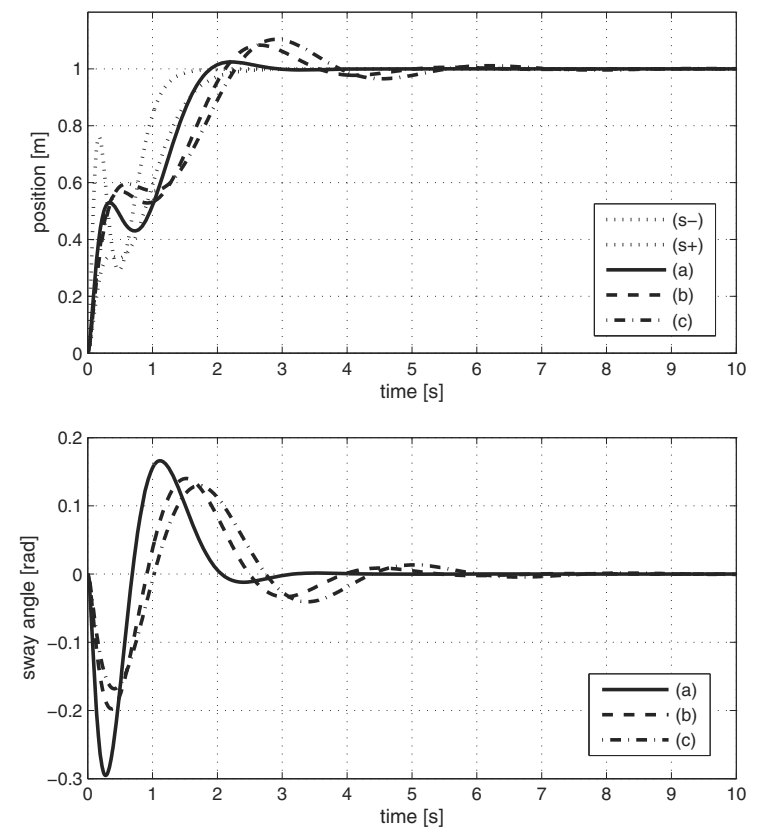

Fig. 8. Crane position and payload sway angle-example of simulations for a TSK controller designed for [1 $\mathrm{m}, 8 \mathrm{~m}$ ], $[10 \mathrm{~kg}, 600 \mathrm{~kg}]$ and $\zeta=0.69$.

lie within the desired interval vector (13) characterizing the acceptable deviation from the nominal point $\left\{a_{1}, b_{2}\right\}$. The coefficients of the characteristic polynomial are close to the right bounds of the vector (13), therefore the response (a) satisfies an acceptable deterioration of the control system performances specified for the nominal point $\left\{a_{1}, b_{2}\right\}$, and the settling time of the response (a)
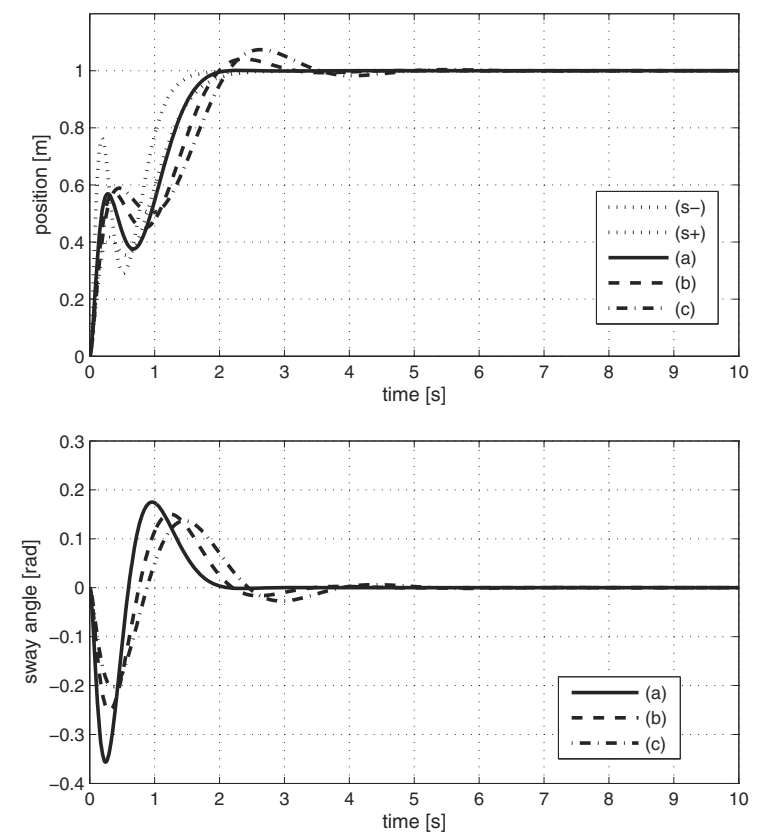

Fig. 9. Crane position and payload sway angle-example of simulations for a TSK controller designed for $[1 \mathrm{~m}, 8 \mathrm{~m}]$, $[10 \mathrm{~kg}, 600 \mathrm{~kg}]$ and $\zeta=0.76$.

is close to the settling time of the response $(\mathrm{s}+)$, despite the overshoot of about 0.02 . In comparison, the responses (b) and (c) are examples of control system performances at the operating point $\left\{\left(a_{1}+a_{2}\right) / 2,\left(b_{1}+b_{2}\right) / 2\right\}$ for $a_{2}=4 \mathrm{~m}$ and $a_{2}=5 \mathrm{~m}$, respectively. The condition (14) is not satisfied at $\left\{\left(a_{1}+a_{2}\right) / 2,\left(b_{1}+b_{2}\right) / 2\right\}$ for any interval vector (13) specifying the acceptable range of the performance deterioration for operating points lying between the nominal points $\left\{a_{1}, b_{1}\right\},\left\{a_{1}, b_{2}\right\},\left\{a_{2}, b_{1}\right\}$ and $\left\{a_{2}, b_{2}\right\}$, which results in arising oscillations and extending the settling time of the responses (b) and (c) in comparison with the response (a).

A similar example of performance comparison for a fuzzy control system designed using the EA for $\zeta=0.76$ assumed for specifying the desired intervals of poles (26) is presented in Fig. 9. The solid line (a) is the system response at the operating point $\left\{\left(a_{1}+a_{2}\right) / 2,\left(b_{2}+b_{3}\right) / 2\right\}$ (where $a_{1}=1.06 \mathrm{~m}, a_{2}=1.85 \mathrm{~m}, b_{2}=334 \mathrm{~kg}$ and $b_{3}=611 \mathrm{~kg}$ ), at which the condition (14) is satisfied for the interval vector (13) representing an acceptable deviation from the nominal point $\left\{a_{1}, b_{3}\right\}$. The settling time of this response is close to that of the response (s+) associated with the right bound of the desired pole interval (26). Moving, e.g., the centre point of the membership function $\mathbf{A}_{2}$ to the right-hand side (Fig. 2) causes a deterioration of closed-loop system performances at the crossover point $\left\{\left(a_{1}+a_{2}\right) / 2,\left(b_{2}+b_{3}\right) / 2\right\}$, which is illustrated in Fig. 11 by the responses (b) and (c) 
Table 3. Results of experiments obtained using the iterative procedure.

\begin{tabular}{|c|c|c|c|c|}
\hline$l$ and $m$ intervals & $\zeta$ & $n_{1}$ & $n_{2}$ & $N=n_{1} \cdot n_{2}$ \\
\hline \hline$[1,8] \mathrm{m}$ & 0.69 & 4 & 3 & 12 \\
{$[10,600] \mathrm{kg}$} & 0.76 & 5 & 3 & 15 \\
\hline$[1,10] \mathrm{m}$ & 0.69 & 5 & 3 & 15 \\
{$[10,1000] \mathrm{kg}$} & 0.76 & 6 & 4 & 24 \\
\hline
\end{tabular}

obtained for the TSK controller with the centre point of the membership function $\mathbf{A}_{2}$ assumed as $a_{2}=3 \mathrm{~m}$ and $a_{2}=4 \mathrm{~m}$, respectively. The condition (14) is not satisfied for $\left\{\left(a_{1}+a_{2}\right) / 2,\left(b_{2}+b_{3}\right) / 2\right\}$, which means that performance deterioration (responses (b) and (c)) exceeds the acceptable range assumed for the operating points lying within the points $\left\{a_{1}, b_{2}\right\},\left\{a_{1}, b_{3}\right\},\left\{a_{2}, b_{2}\right\}$, $\left\{a_{2}, b_{3}\right\}$.

The results obtained using the EA (Table 1) were compared with those of the iterative method employed to design a TSK fuzzy controller. In the iterative method, the rope length $\left[l^{-}, l^{+}\right]$and payload mass $\left[m^{-}, m^{+}\right]$ ranges were divided into even intervals (respectively, 0.1 $\mathrm{m}$ and $10 \mathrm{~kg}$ ). In the two-stage iterative procedure, starting from $l_{i}=l^{-}$and $m_{j}=m^{-}, i$ and the next $j$ are incremented to find the minimum number of membership function midpoints between the lower and upper bounds of intervals $\left[l^{-}, l^{+}\right]$and $\left[m^{-}, m^{+}\right]$. At each iteration, $l_{i}$ and $m_{j}$ are assumed as the temporary center points $\left\{a_{i}, b_{j}\right\}$ of membership functions, which leads to creating temporary fuzzy rules with the parameters of controllers $\mathbf{K}_{k}$ determined in their conclusions according to system (11).

The condition (14) is tested for the crossover points of memberships functions. If it is satisfied, the temporarily fuzzy sets and rules are removed. If the condition (14) is violated at a sample point $l_{i}$ or $m_{j}$, a new fuzzy set is created with a point of a membership function at $l_{i-1}$ or $m_{j-1}$, respectively. Creating Tables 3 and 4 present the results of this procedure applied to design a TSK fuzzy controller for the same conditions which were assumed during experiments conducted using the EA. The method does not lead to obtaining an optimal solution due to assuming the initial set of membership functions with midpoints at the bounds of intervals $\left[l^{-}, l^{+}\right]$and $\left[\mathrm{m}^{-}, \mathrm{m}^{+}\right]$before starting an iterative process. Therefore, in the two cases, for the parameter intervals $[1 \mathrm{~m}, 8 \mathrm{~m}]$ and $[10 \mathrm{~kg}, 600 \mathrm{~kg}]$, and $[1 \mathrm{~m}, 10 \mathrm{~m}]$ and $[10 \mathrm{~kg}, 1000$ $\mathrm{kg}$ ] (Table 3), the iterative procedure resulted in obtaining a larger size of RB: 12 and 24 rules, respectively.

\section{Conclusions}

The hybrid method combining the evolutionary-based search strategy, interval mathematics and pole assignment-based closed-loop control synthesis was
Table 4. Parameters of membership functions determined using the iterative procedure.

\begin{tabular}{|c|c|c|}
\hline $\begin{array}{l}l \text { and } m \text { in- } \\
\text { tervals }\end{array}$ & $\zeta$ & $\begin{array}{l}\text { parameters of membership func- } \\
\text { tions }\end{array}$ \\
\hline $\begin{array}{l}{[1,8] \mathrm{m},} \\
{[10,600] \mathrm{kg}}\end{array}$ & 0.69 & $\begin{array}{l}\mathbf{a}=[1.0, \\
\mathbf{b}=[10,520,600]\end{array}$ \\
\hline $\begin{array}{l}{[1,8] \mathrm{m},} \\
{[10,600] \mathrm{kg}}\end{array}$ & 0.76 & 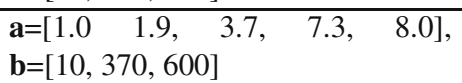 \\
\hline$[1,10] \mathrm{n}$ & 0.69 & 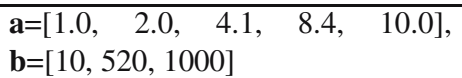 \\
\hline $\begin{array}{l}{[1,10] \mathrm{m},} \\
{[10,1000] \mathrm{kg}}\end{array}$ & 0.76 & 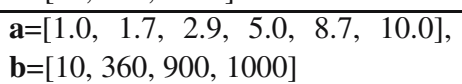 \\
\hline
\end{tabular}

proposed in this paper to design a TSK fuzzy controller. Closed-loop system performance conditions are derived from the interval Diophantine equation and applied to define the objective function of the evolutionary algorithm used to optimize the number of fuzzy sets on inputs of a TSK fuzzy controller. The proposed method was implemented to design an anti-sway crane control system robustness to the rope length and mass of a payload variation.

The evolutionary-based strategy used to optimize the design process of a fuzzy controller provides effective reproductions techniques for searching the solution space in $\mathrm{RB}$ optimization through minimizing the numbers of fuzzy sets determined for input variables. The hybridization of arithmetical crossover, uniform and non-uniform mutation, and deletion/insertion mutation ensures the diversity of genome sizes, as well as diversity in the parameter space of rule antecedents. The recombination mechanism ensures fine exploration of the best promising regions of the possible solution space by tuning the triangular-shaped membership function parameters.

The results of experiments conducted for different conditions assumed for system parameter intervals confirm that the developed method of robust fuzzy controller synthesis allows designing a TSK controller placing the coefficients of a closed-loop characteristic polynomial within the desired intervals. The paper also describes an iterative procedure of designing a TSK controller. Both the methods, the EA and the iterative procedure, allows designing the robust fuzzy controller. However, the results of experiments conducted for different conditions assumed for desired closed-loop system performances proved that the EA results in a smaller number of fuzzy sets and rules of a TSK controller required to satisfy the acceptable range of closed-loop system performance deterioration specified in the form of desired intervals of closed-loop characteristic polynomial coefficients. 


\section{Acknowledgment}

I wish to thank the anonymous referees for their thorough review, comments and suggestions, which significantly helped to improve the quality of this contribution.

\section{References}

Auernig, J.W. and Troger, H. (1987). Time optimal control of overhead cranes with hoisting of the load, Automatica 23(4): 437-447.

Bańka, S., Dworak, P. and Jaroszewski, K. (2013). Linear adaptive structure for control of a nonlinear MIMO dynamic plant, International Journal of Applied Mathematics and Computer Science 23(1): 47-63, DOI: 10.2478/amcs-2013-0005.

Benhidjeb, A. and Gissinger, G.L. (1995). Fuzzy control of an overhead crane performance comparison with classic control, Control Engineering Practice 3(12): 1687-1696.

Chang, C.-Y. (2006). The switching algorithm for the control of overhead crane, Neural Computing and Applications 15(3-4): 350-358.

Chang, C.-Y. (2007). Adaptive fuzzy controller of the overhead crane with nonlinear disturbances, IEEE Transactions on Industrial Informatics 3(2): 164-172.

Chapellat, H., Keel, L.H. and Bhattacharyya, S.P. (1994). External robustness properties of multilinear interval systems, Automatica 30(6): 1037-1042.

Dahleh, M., Tesi, A. and Vicino, A. (1993). An overview of extremal properties for robust control of interval plants, Automatica 29(3): 707-721.

De Jong, K.A., Spears, W.M. and Gordon, D.F. (1993). Using genetic algorithms for concept learning, Machine Learning 13(2-3): 161-188.

Fang, Y., Ma, B., Wang, P. and Zhang, X. (2012). A motion planning-based adaptive control method for an underactuated crane system, IEEE Transactions on Control Systems Technology 20(1): 241-248.

Filipic, B., Urbancic, T. and Krizman, V. (1999). A combined machine learning and genetic algorithm approach to controller design, Engineering Applications of Artificial Intelligence 12(4): 401-409.

Hsu, C.-C., Chang, S.-C. and Yu, C.-Y. (2007). Tolerance design of robust controllers for uncertain interval systems based on evolutionary algorithms, IET Control Theory and Applications 1(1): 244-252.

Hyla, P. (2012). The crane control systems: A survey, Proceedings of the 17th IFAC International Conference on Methods and Models in Automation and Robotics MMAR, Międzyzdroje, Poland, pp. 505-509.

Kang, Z., Fujii, S., Zhou, C. and Ogata, K. (1999). Adaptive control of a planar gantry crane by the switching of controllers, Transactions of Society of Instrument and Control Engineers 35(2): 253-261.
Karajgikar, A., Vaughan, J. and Singhose, W. (2011). Double-pendulum crane operator performance comparing pd-feedback control and input shaping, Proceedings of the ECCOMAS Thematic Conference on Advances in Compuational Multibody Dynamics, Brussels, Belgium, pp. 1-14.

Kharitonov, V.L. (1978). Asymptotic stability of an equilibrium position of a family of systems of linear differential equations, Differential'nye Uravneniya 14(11): 2086-2088.

Kijima, Y., Ohtsubo, R., Yamada, S. and Fujikawa, H. (1995). An optimization of fuzzy controller and it's application to overhead crane, Proceedings of the IEEE IECON 21st International Conference on Industrial Electronics, Control, and Instrumentation, Tokyo, Japan, pp. 1508-1513.

Kimiaghalam, B., Homaifar, A., Bikdash, M. and Dozier, G. (1999). Genetic algorithms solution for unconstrained optimal crane control, Proceedings of the IEEE Congress on Evolutionary Computation, Washington, DC, USA, pp. 2124-2130.

Kimiaghalan, B., Homaifar, A., Bikdash, M. and Sayyarrodsari, B. (2002). Genetic algorithm based gain scheduling, Proceedings of the Congress on Evolutionary Computation, Greensboro, NC, USA, pp. 540-545.

Kluska, J. (2006). Transformation lemma on analytical modeling via Takagi-Sugeno fuzzy system and its applications, 8th International Conference on Artificial Intelligence and Soft Computing (ICAISC 2006), Zakopane, Poland, pp. 230-239.

Kluska, J. (2009). Analytical Methods in Fuzzy Modeling and Control, Studies in Fuzziness and Soft Computing, Vol. 241, Springer-Verlag, Berlin/Heidelberg.

Lee, C.-H., Lee, Y.-H. and Teng, C.-C. (2002). A novel robust PID controllers design by fuzzy neural network, Proceedings of the American Control Conference, Anchorage, AK, USA, pp. 1561-1566.

Li, X. and Yu, W. (2012). Anti-swing control for an overhead crane with fuzzy compensation, Intelligent Automation and Soft Computing 18(1): 1-11.

Liu, D., Yi, J. and Tan, M. (2002). Proposal of GA-based two-stage fuzzy control of overhead crane, Proceedings of the IEEE Conference on Computers, Communications, Control and Power Engineering, Beijing, China, pp. 1721-1724.

Liu, D., Yi, J., Zhao, D. and Wang, W. (2005). Adaptive sliding mode fuzzy control for a two-dimensional overhead crane, Mechatronics 15(5): 505-522.

Mahfouf, M., Kee, C.H., Abbod, M.F. and Linkens, D.A. (2000). Fuzzy logic-based anti-sway control design for overhead cranes, Neural Computing and Applications 9(1): 38-43.

Mallan, S., Milanese, M. and Taragna, M. (1997). Robust analysis and design of control systems using interval arithmetic, Automatica 33(7): 1363-1372.

McNichols, H. and Fadali, M.S. (2003). Selecting operating points for discrete-time gain scheduling, Computers and Electrical Engineering 29(2): 289-301. 
Mendez, J.A., Acosta, L., Moreno, L., Torres, S. and Marichal, G.N. (1999). An application of a neural self-tuning controller to an overhead crane, Neural Computing and Applications 8(2): 143-150.

Michalewicz, Z. and Janikow, C.Z. (1991). Handling constraints in genetic algorithm, Proceedings of the 4th International Conference on Genetic Algorithms, San Diego, CA, USA, pp. 151-157.

Moon, M.S., VanLandingham, H.F. and Beliveau, Y.J. (1996). Fuzzy time optimal control of crane load, Proceedings of the 35th Conference on Decision and Control, Kobe, Japan, pp. 1127-1132.

Moore, R. (1966). Interval Analysis, Prentice-Hall, Englewood Cliffs, NJ.

Moustafa, K. A. F. (2001). Reference trajectory tracking of overhead cranes, Journal of Dynamic Systems, Measurement, and Control 123(1): 139-141.

Nakazono, K., Ohnisihit, K. and Kinjot, H. (2007). Load swing suppression in jib crane systems using a genetic algorithm-trained neuro-controller, Proceedings of the International Conference on Mechatronics, Kumamoto, Japan, pp. 1-4.

Oh, S.-K., Pedrycz, W., Rho, S.-B. and Ahn, T.-C. (2004). Parameter estimation of fuzzy controller and its application to inverted pendulum, Engineering Applications of Artificial Intelligence 17(1): 37-60.

Sadati, N. and Hooshmand, A. (2006). Design of a gain-scheduling anti-sway controller for tower cranes using fuzzy clustering techniques, Proceedings of the International Conference on Computational Intelligence for Modeling, Control and Automation, Sydney, Australia, p. 172.

Sakawa, Y. and Shindo, Y. (1982). Optimal control of container cranes, Automatica 18(3): 257-266.

Singer, N., Singhose, W. and Kriikku, E. (1997). An input shaping controller enabling cranes to move without sway, Proceedings of the American Nuclear Society 7th Topical Meeting on Robotics and Remote Systems, Augusta, GA, USA, pp. 225-231.

Smalko, Z. and Szpytko, J. (2009). Safety in engineering practice, Proceedings of the 17th European Safety and Reliability Conference ESREL, Valencia, Spain, pp. 1231-1237.

Smith, S.F. (1980). A Learning System Based on Genetic Adaptive Algorithms, Ph.D. thesis, University of Pittsburgh, Pittsburgh, PA.

Smoczek, J. and Szpytko, J. (2008). A mechatronics approach in intelligent control systems of the overhead traveling cranes prototyping, Information Technology and Control 37(2): 154-158.

Smoczek, J. and Szpytko, J. (2010). Fuzzy logic approach to the gain scheduling crane control system, Proceedings of the 15th IFAC International Conference on Methods and Models in Automation and Robotics MMAR, Międzyzdroje, Poland, pp. 261-266.
Smoczek, J. and Szpytko, J. (2011). Design of a fuzzy gain scheduling controller for the anti-sway crane system, Proceedings of the 26th ISPE International Conference on CAD/CAM, Robotics and Factories of the Future, CARSFOF, Kuala Lumpur, Malaysia, pp. 809-818.

Solihin, M.I., Wahyudi and Legowo, A. (2010). Fuzzy-tuned antiswing control of automatic gantry crane, Journal of Vibration and Control 16(1): 127-145.

Sugeno, M. and Kang, G.T. (1988). Structure identification of fuzzy model, Fuzzy Sets and Systems 28(1): 15-33.

Szpytko, J. and Wozniak, D.A. (2007). To keep operational potential of transport device e-based on reliability indicators, Proceedings of the European Safety and Reliability Conference ESREL, Stavanger, Norway, pp. 2377-2384.

Takagi, T. and Sugeno, M. (1985). Fuzzy identification of systems and its application to modeling and control, IEEE Transactions on Systems, Man and Cybernetics 15(1): 116-132.

Trabia, M.B., Renno, J.M. and Moustafa, K.A.F. (2008). Generalized design of an anti-swing fuzzy logic controller for an overhead crane with hoist, Journal of Vibration and Control 14(3): 319-346.

Warmus, M. (1956). Calculus of approximations, Bulletin de l'Academie Polonaise des Sciences IV(5): 253-259.

Yi, J., Yubazaki, N. and Hirota, K. (2003). Anti-swing and positioning control of overhead traveling crane, Information Sciences 155(1-2): 19-42.

Young, R.C. (1931). The algebra of many-valued quantities, Mathematische Annalen 104(1): 260-290.

Yu, W., Moreno-Armendariz, A. and Rodriguez, F.O. (2011). Stable adaptive compensation with fuzzy CMAC for an overhead crane, Information Sciences 181(21): 4895-4907.

Zubowicz, T. and Brdyś, M.A. (2013). Stability of softly switched multiregional dynamic output controllers with a static antiwindup filter: A discrete-time case, International Journal of Applied Mathematics and Computer Science 23(1): 65-73, DOI: 10.2478/amcs-2013-0006.

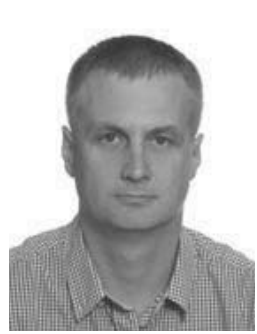

Jarosław Smoczek received the M.Sc. in automatics and metrology and the Ph.D. in machine construction and utilization in 1999 and 2003, respectively. Currently he is an assistant professor at the Faculty of Mechanical Engineering and Robotics, AGH University of Science and Technology in Kraków. He has authored over 80 papers and two books. His current research interests include automation and exploitation of material handling devices, computational intelligence, control systems, supervisory control, data acquisition and visualization.

Received: 7 May 2013 Revised: 10 July 2013 